\title{
Designing a Microstrip coupled line bandpass filter
}

\author{
Ragani Taoufik*, N. Amar Touhami, M. Agoutane \\ Electronics, Instrumentation and Microwave Laboratory, University Abdelmalek Essaadi, Faculty of Science, \\ Street M'hannech II B.P 2121, Tétouan, Morocco \\ *Corresponding author E-mail: Regani.taoufik@gmail.com
}

\begin{abstract}
Bandpass filters play a significant role in wireless communication systems. Transmitted and received signals have to be filtered at a certain center frequency with a specific bandwidth, in this paper, a coupled-line bandpass Filter at the center frequency $6 \mathrm{GHz}$ with the wide bandwidth of $2 \mathrm{GHz}$. this type of filter can be used in WLAN and other applications for the frequency range of 5-7 GHz.
\end{abstract}

Keywords: BANDPASS FILTERS, COUPLED LINE, CST 2011, WLAN

\section{Introduction}

The broadband wireless access (BWA) is an important issue in current developments of the modern wireless communication system. To meet this trend, the bandpass filters with relatively wide bandwidth are frequently required in the RF front ends. In microwave communication systems, the bandpass filter is an essential component, which is usually used in both receivers and transmitters [1].In this work we would like to give a way to design a bandpass filter for the WLAN application at the frequency $6 \mathrm{GHz}$ with parallel-coupled Microstrip.

The design goals and parameters have been shown in the Table 1.

Table 1: Design Goals of the Filter

\begin{tabular}{lc} 
& \\
\hline Ordre of the Filtre & 3 \\
Frequency & $6 \mathrm{GHz}$ \\
$\varepsilon_{r}$, FR4 & 4.3 \\
Height of the Substrate & $1.56 \mathrm{~mm}$ \\
Thickness of the conducting & $0.035 \mathrm{~mm}$ \\
Loss tangent & 0.025 \\
Fractional Bandwidth & $33 \%$ at $6 \mathrm{GHz}$ \\
Bandwidth & $>1 \mathrm{GHz}$ \\
\hline
\end{tabular}

\section{Parallel Coupled lines Bandpass Filter}

Fig.1 gives the circuit implementation of the filter by means of concentrated components like inductors (L) and capacitors (C), for the even and odd filter degree (n). 

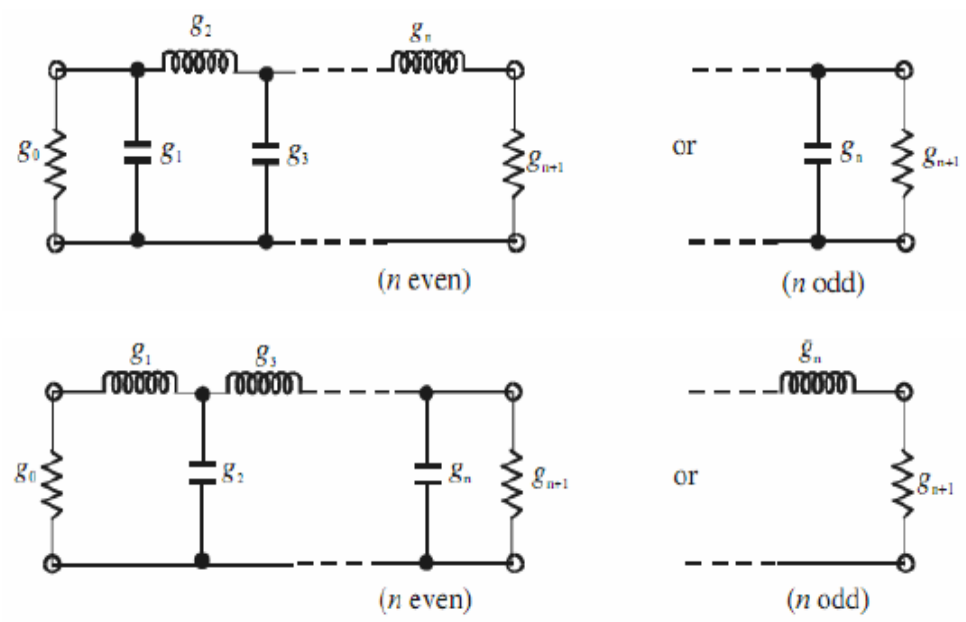

Fig.1: Realization of filter using LC components.

The component values can be calculated with the following rules:

$$
\begin{aligned}
& \mathrm{g}_{0}=1 \\
& \mathrm{~g}_{1}=\frac{2}{\gamma} \sin \left(\frac{\pi}{2 \mathrm{n}}\right) \\
& \mathrm{g}_{\mathrm{i}}=\frac{1}{\mathrm{~g}_{\mathrm{i}-1}}\left(\frac{4 \sin \left(\frac{(2 \mathrm{i}-1) \pi}{2 \mathrm{n}}\right) \sin \left(\frac{(2 \mathrm{i}-3) \pi}{2 \mathrm{n}}\right)}{\gamma^{2}+\sin ^{2}\left(\frac{(\mathrm{i}-1) \pi}{\mathrm{n}}\right)} \sin \left(\frac{(2 \mathrm{i}-1) \pi}{2 \mathrm{n}}\right)\right)
\end{aligned}
$$

for $\mathrm{i}=2$ to $\mathrm{n}$

$$
g_{n+1}=\left\{\begin{array}{ll}
1 & \text { for odd } n \\
\hline \cot ^{2}\left(\frac{\beta}{4}\right) & \text { for even } n
\end{array}\right\}
$$

Where $\quad \beta=\operatorname{Ln}\left[\cot ^{2}\left(\frac{L_{A, r}}{17.37}\right)\right]$ and $\gamma=\sin ^{-1}\left(\frac{\beta}{2 n}\right)$

\subsection{Designing bandpass filter}

Figure 2 shows the filter structure observed in this work. This filter type is known as parallel-coupled filter. The strips are arranged parallel close to each other, so that they are coupled with certain coupling factors. We use the following equations for designing the parallel-coupled filter [2].

$$
\begin{aligned}
& \frac{J_{0,1}}{Y_{0}}=\sqrt{\left[\frac{\pi F B W}{2 g_{0} g_{1}}\right]} \\
& \frac{J_{j ; j+1}}{Y_{0}}=\frac{[\pi F B W]}{\left[2 \sqrt{g_{j} g_{j+1}}\right]}
\end{aligned}
$$

$$
\text { For } \mathrm{j}=1 \text { to } \mathrm{n}=1 \quad \frac{J_{n, n+1}}{Y_{0}}=\sqrt{\left[\frac{\pi F B W}{2 g_{n} g_{n+1}}\right]}
$$

FBW is the relative bandwidth as explained before, $J_{j ; j+1}$ is the characteristic admittance of $\mathrm{J}$ inverter and $Y_{0}$ is the characteristic admittance of the connecting transmission line. 
With the data of characteristic admittance of the inverter, we can calculate the characteristic impedances of even-mode and odd-mode of the parallel-coupled Microstrip transmission line, as follows [2, 3].

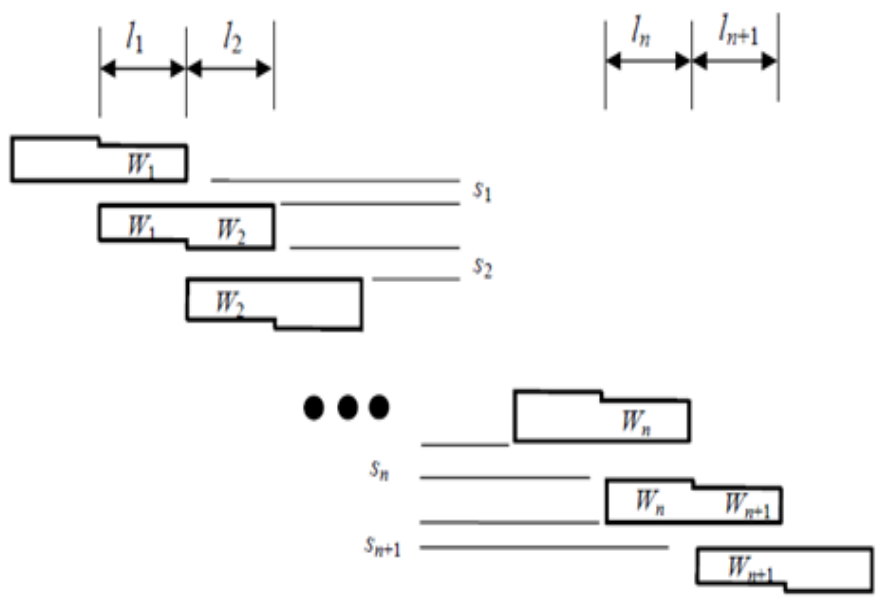

Fig 2: Parallel Bandpass Filter

for $\mathrm{j}=0$ to $n$

and

$$
\left(Z_{o e}\right)_{j, j+1}=\left(\frac{1}{Y_{0}}\right)\left[1+\left(\frac{J_{j ; j+1}}{Y_{0}}\right)+\left(\frac{J_{j ; j+1}}{Y_{0}}\right)^{2}\right]
$$

for $\mathrm{j}=0$ to $n$

$$
\left(Z_{o 0}\right)_{j, j+1}=\left(\frac{1}{Y_{0}}\right)\left[1-\left(\frac{J_{j ; j+1}}{Y_{0}}\right)+\left(\frac{J_{j ; j+1}}{Y_{0}}\right)^{2}\right]
$$

From these values, width, length and spacing of the parallel coupled line are calculated and are shown in Table-2,

\begin{tabular}{cccccccc}
\multicolumn{8}{c}{ Table 2: Specifications of Parallel coupled Microstrip Lines } \\
\hline $\mathrm{n}$ & $g_{n}$ & $Z_{0} J_{n}$ & $Z_{o e}(\Omega)$ & $Z_{o o}(\Omega)$ & $\mathrm{W}(\mathrm{mm})$ & $\mathrm{L}(\mathrm{mm})$ & $\mathrm{S}(\mathrm{mm})$ \\
\hline 1 & 0.6292 & 0.63 & 101.5 & 38.5 & 1.4 & 6 & 0.2 \\
2 & 0.9703 & 0.32 & 71 & 39 & 2.4 & 6 & 0.2 \\
3 & 0.6292 & 0.32 & 71 & 39 & 2.4 & 6 & 0.2 \\
4 & 1.0 & 0.63 & 101.5 & 38.5 & 1.4 & 6 & 0.2 \\
\hline
\end{tabular}

\section{Design configuration and simulated results}

\subsection{Geometry on simulator}

In this proposed design the height of the substrate is $1.5 \mathrm{~mm}$ and relative permittivity 4.3 and the conductor thickness $0.035 \mathrm{~mm}$ and loss tangent is 0.025 , figure 3 shows the 3 -dimensional view of proposed bandpass filter. Proposed design is simulated in CST microwave studio 2011 [5]. 


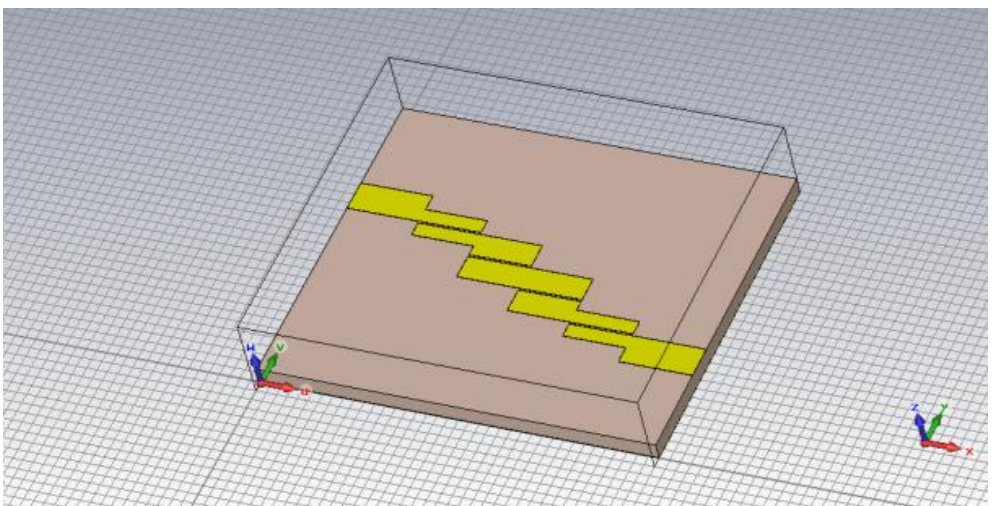

Fig 3: Geometry of Microstrip Band Pass Filter

\subsection{Simulation result}

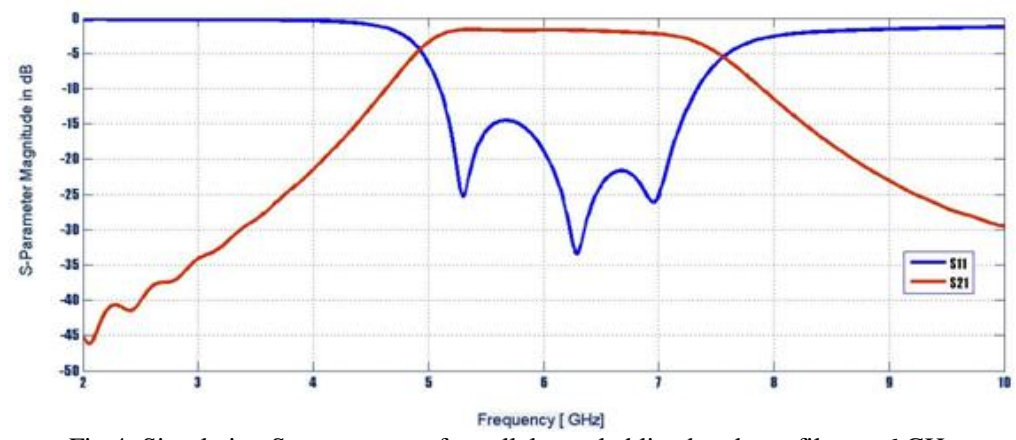

Fig 4: Simulation S-parameters of parallel coupled line bandpass filter at $6 \mathrm{GHz}$

Fig.4 shows the simulation response of conventional parallel-coupled microstrip line bandpass filter with centre frequency $6 \mathrm{GHz}$.

\section{Conclusion}

Designing of bandpass filter with Chebyshev approach in combination with concentrated components, i.e. inductors and capacitors and its computational verification in form of parallel coupled microstrip lines with the CST give very good filter characteristics at the center frequency $6 \mathrm{GHz}$ with frequency bandwidth of about $2 \mathrm{GHz}$ as required at the specification stage. At the center frequency the insertion loss and reflection factor has the values about $-2 \mathrm{~dB}$ and better than $-15 \mathrm{~dB}$, respectively.

\section{References}

[1] D.M. Pozar, "Microwave engineering", 2nd edition, 1998 John-Wiley \& Sons.

[2] J.S. Hong, and M.J. Lancaster, Microstrip Filters for RF/Microwave Applications, Wiley, New York, 2001

[3] M. Kirschning, and R.H. Jansen, "Accurate wide-range design equations for parallel coupled microstrip lines", IEEE Trans. MTT-32, Jan. 1984, pp. 83-90. Corrections in IEEE Trans. MTT-33, March 1985, p. 288.

[4] R..Mongia, P. Bhartia and I.J.Bahl, RF and Microwave Coupled-line Circuits, 2nd ed., Artech House, Boston, 2007

[5] CST Microwave Studio 2011 